\title{
Telerobotics for hang-up assessment and removal - an idea whose time has come
}

\author{
G Baiden Penguin Automated Systems Inc., Canada
}

\begin{abstract}
The removal of rock blockages in drawpoints in mining operations has long been a risky undertaking, especially in high overhead conditions. In fact, the assessment of where to place those charges has been difficult due to the lack of visibility and safe access to the area. Thus, the traditional practise of the wedging of long poles with explosives on them, keeping the operator in as safe a location as possible, has been one of the tried and true methods in attempting to remove blockages. Unfortunately, this is less than perfect, usually taking several attempts to achieve the blockage removal. Each successive attempt can result in an even more dangerous condition for the worker and/or damage to the mine, often due to successively larger amounts of explosives.

Over the years, different ideas have been attempted to change this process and reduce the inherent safety risks. Some of the technologies that have been attempted ranged from projectiles fired at the hang-up, to large, cumbersome arms, to massive amounts of explosives, each with their own safety risks resulting in dangerous situations for the miner and the mine.

We have reached a point where combining mining problems with gaming technology and robots can achieve results that the mining industry has struggled to achieve so far. In this case, the safe assessment and removal of hang-ups and oversize could be possible by combining Penguin's proprietary GPS-denied positioning system with a six-degree of freedom telerobot, virtual gaming and teleoperation over a wireless network. Penguin Automated Systems has developed a robot system capable of working in a rock blockage safely.

The idea draws on the latest in telerobotics technology combined with $3 D$ scanning and underground geospatial positioning. The hang-up removal is performed by scanning the inside of the hang-up to rapidly develop a geospatially 3D model. The 3D model enables the picking of the 'keystone'. The robot arm on the robot then uses this $3 D$ virtual model of the robot system and the drawpoint to locate and load the hole to clear the hang-up.
\end{abstract}

Keywords: hang-up, telerobotic, blasting, assessment, removal

\section{Cave mining and the issue of hang-ups}

Cave mining is one of the major methods employed by mining companies today. Cave mining, performed properly with the right orebody, is the lowest cost underground mining method. There are many parameters to make a low-cost cave mine work according to Laubscher (1994) and Chitombo (2010). These parameters include cavability, drawpoint spacing, draw control, dilution entry, layouts, undercutting, ground support and fragmentation. Most importantly, caving operation depends on size distribution as discussed by Laubscher (1994). Rock blockages or hang-ups in underground drawpoints and drawpoint structures are typically caused by oversize issues and the arching of rocks to form a blockage. This makes caving dependent on the rapid, safe removal of hang-ups and oversize. Rock blockages due to oversize and hang-ups create and/or cause many problems in all forms of mining. Production rock blockages cause dilution and recovery problems if not dealt with promptly. Furthermore, the removal of these blockages, regardless of type, create potential safety issues for those involved.

This paper was originally published in The AusIMM Bulletin, February 2017. Acknowledgement is given to The Australasian Institute of Mining and Metallurgy for their permission to republish this paper. 


\section{What does a hang-up assessment and removal robotic system need to do?}

The Hang-up Assessment and Removal Robotic System safely allows any operator to assess and remove rock blockages that are hung-up from a safe location. The Hang-up Assessment and Removal Robot System was envisaged to do two very important tasks:

- Assess a blockage or hang-up to understand the nature of the problem.

- Determine how to deal with the problem.

The idea draws on the latest in telerobotics technology combined with 3D scanning and underground geospatial positioning. The hang-up removal is performed by scanning the inside of the hang-up to rapidly develop a geospatially placed 3D model for the operator to use. The 3D model is detailed enough to attempt to pick the 'keystone'. A blasting engineer or the operator can then determine the exact position where the explosive charge should be placed in the 3D model and thus, the actual hang-up. The robot arm on the robot controlled by the operator then uses this $3 \mathrm{D}$ virtual reality model of the robot system and the actual drawpoint information model to display this location information. At this point, the robot arm reaches into the hang-up to position the charge using the kinematic model of the robot system. The 3D model is then used to provide views inside the hang-up for the operator to move the arm. This process allows the operator to place the charge within the 3D representation of the drawpoint from a long distance away. As the process continues, the end of the arm, with a 3D vision system, drills the rock, and the explosives are loaded precisely. The robot then returns the blasting cable to the command station located in a truck a safe distance away from the blockage.

As can be seen in Figure 1, entering past the drawpoint collar can be an unsafe task. The potential for rock to fall and roll out the collar is high. Therefore, current visual assessment of the hang-up can only be done from the side of the collar of the drawpoint allowing for a partial view at best. That technique then uses concussion charges on poles, gradually increasing their size until the hang-up is removed. This typically solves the problem but continuously puts the operator at risk and usually causes damage to the mine. For years there has not been a better practice due to the difficult nature of the problem.

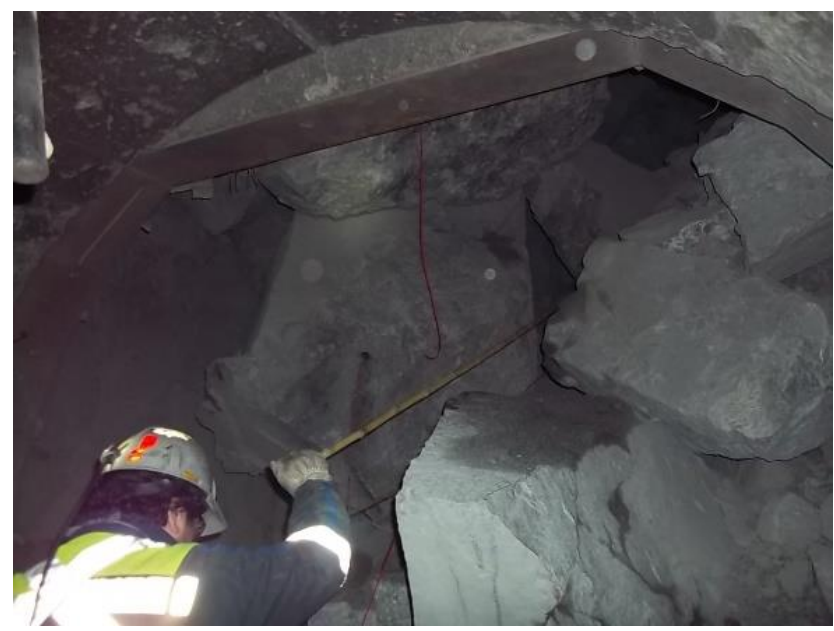

Figure 1 Current practice of a miner inserting explosives into low hang-up

In changing this process, Penguin's GPS-denied positioning system, demonstrated at Codelco, proved that a better way would be to assess the hang-up using optical and laser scanning techniques, creating a 3D model of the inside of the hang-up. This model would be used as a means for the operator, or blasting engineer, to determine where to place a charge for maximum effectiveness while protecting the operator and reducing damage to the opening. Moreover, if the exact position of the 3D model could be matched with the machine position, it would be possible to reach a robotic arm to the exact location needed to drill, load and blast the charge to shatter the keystone, or substantially move it, to free the hang-up. 
So, in general, what would it take to accomplish a system such as this for removing hang-ups? The main need was to create a rugged, mathematical machine that could be telerobotically operated with accurate position control and a communication system capable of moving multiple video, scanners and data streams in a closed loop fashion with the machine and arm control. During the initial stages of creating this system, several of the technologies seemed to be unavailable for underground mining. In particular, the technologies for accurate real-time underground positioning and high-bandwidth networking appeared unavailable.

The first task to complete is the assessment function. Assessment requires the creation of a 3D model of the hang-up (Figure 2). Today miners can only get a limited view of a hang-up due to the difficulty of looking into a drawpoint. Since a drawpoint is basically an open flow of rock, personnel should not enter the area to assess the hang-up. With a robot, this assessment process is not a problem, because the robot can enter the drawpoint if absolutely necessary. Therefore, a clearer picture can be captured to gather point cloud data of the hang-up, giving a much superior look into the drawpoint. The patented subsystem consists of a subsurface positioning system and a kinematic model of the machine and robot arms to support mathematical measuring and imaging of the hang-up.

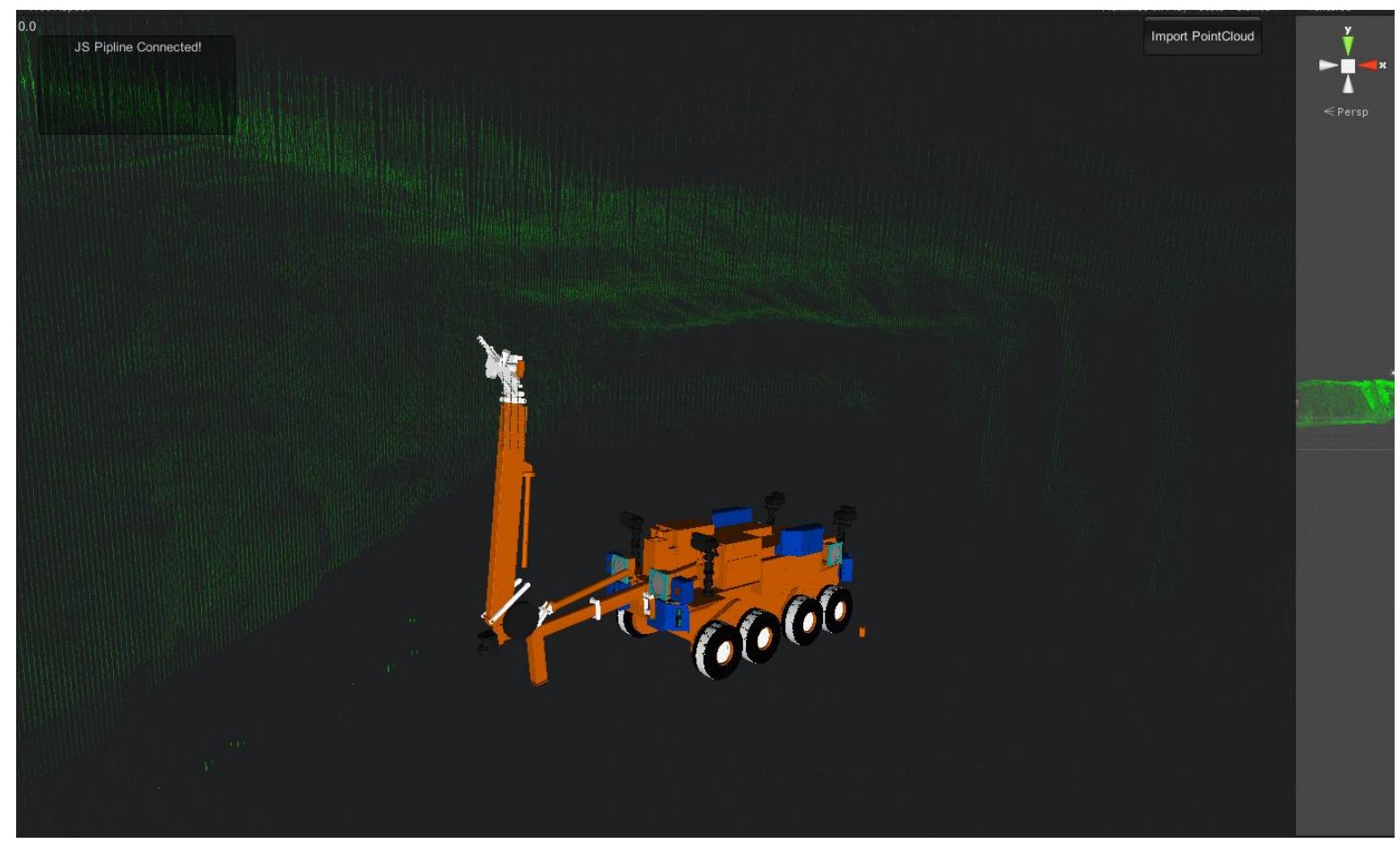

Figure 2 3D scanned model of the mining of an area with an operational model of the hang-up robot that emulates the real hang-up robot

With the image generated at the assessment stage, the robot needs to position and extend the lower and upper arm sections to get the drill and explosive to the precise location determined by the experience of the blasting engineer and operator. Once positioned, the location is drilled and readied for explosive insertion and pulling the detonator initiation wire back to the centre of the drift. The detonator initiation wire will be connected to allow multiple blasts at cross-shift. The robot drills and inserts a charge each deployment.

The development and patenting of a new optical communication network made this concept feasible as its capability far exceeded current best performance in radio networking. This provided communication network supported for teleoperation control of an assessment system with scanners, cameras and data control, and allowed the creation of a precise robot arm and mobile delivery system. What the data transfer system could then support was the kinematic modelling of a six degree of freedom robot with an arm, and an end effector consisting of a rock drill and explosives loading device. Thus, a currently dangerous task could be performed, provided that a mobile computer numerically controlled (CNC) machine with accurate position control was established. 


\section{$3 \quad$ Hang-up robot system}

The hang-up robot (Figure 3$)$ is a 5,530 $\mathrm{kg}(12,191 \mathrm{lbs})$ all-electric machine consisting of the undercarriage, power system, two-stage robot arm, hang-up scanning system, end effectors, camera system, control system, wireless networking, safety system and diagnostic system. While the system has been especially designed for the Hang-up Assessment and Removal Robot System, the end effectors can be modified to handle any number of power tools.

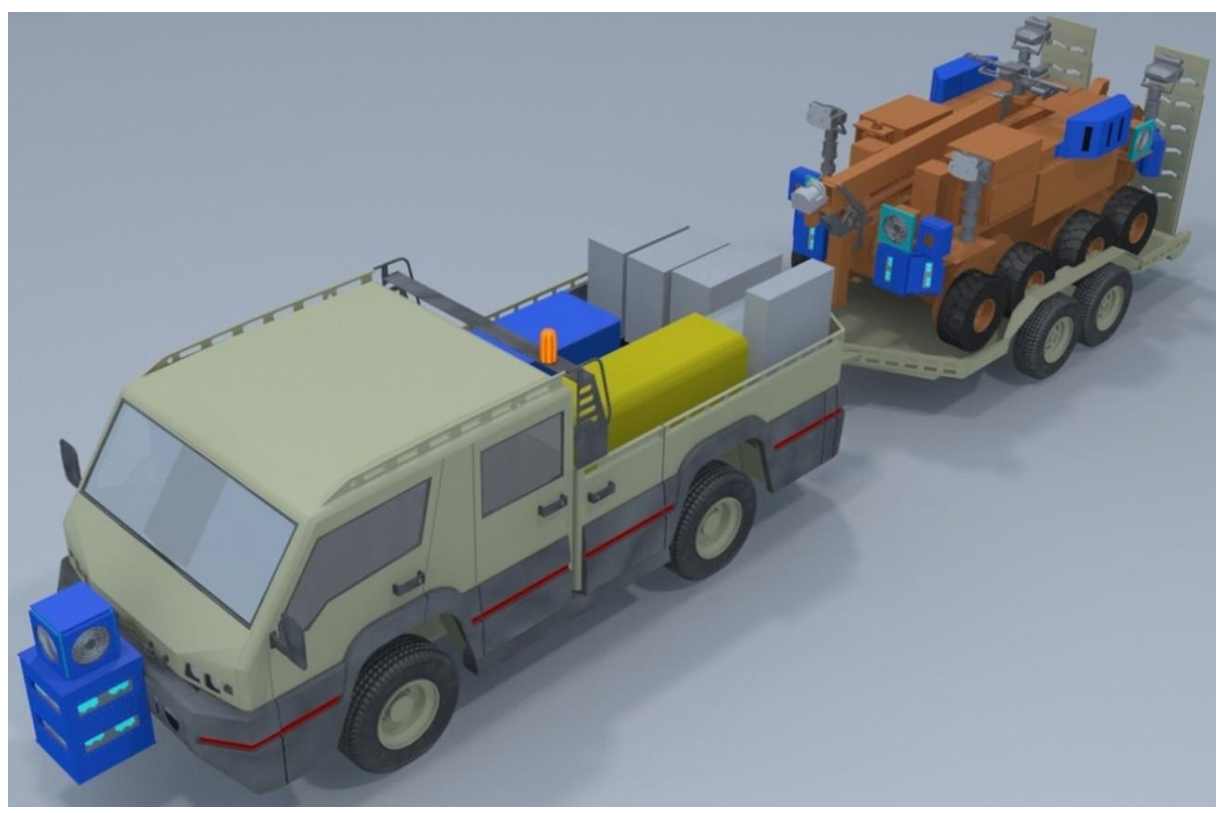

Figure 3 Artist's concept of the telerobotic Hang-up Assessment and Removal System

The system (Figures 4 and 5) consists of:

- A Normet RBO housing a telecommand centre, hydraulic generator, robot battery charger and explosive transportation container.

- The telecommand centre housed in a Normet RBO truck is wirelessly connected to the robot using radio networking and optical techniques.

- A geospatially operated robot and high reach two-stage arm capable of reaching into the hang-up and building a 3D image of the hang-up to assess the optimal means to remove a hang-up.

- An end effector for drilling and loading explosives into the hang-up to blast the rock blockages imaged in the 3D scans from the assessment.

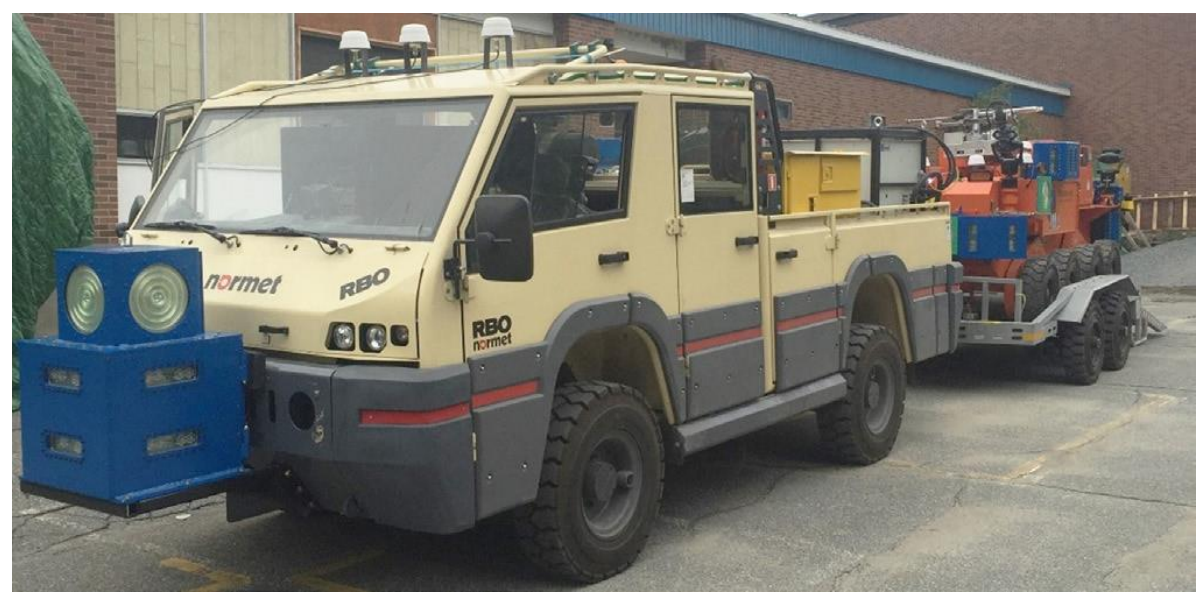

Figure 4 Telerobotic Hang-up Assessment and Removal System 


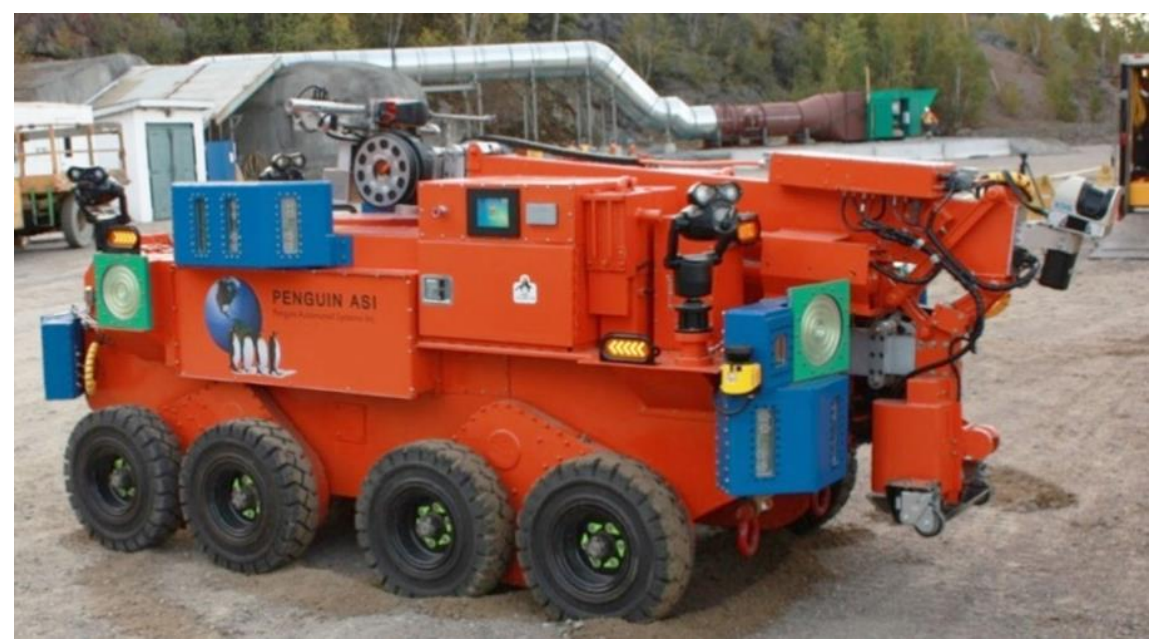

Figure 5 Hang-up telerobot

Some additional features of the system are described in the following sections.

\subsection{Robot undercarriage}

The robot undercarriage is a powerful all-electric machine. The unit is a totally waterproof tub with sealed external gearboxes for operation in difficult environments with gradeability of $30^{\circ}$. The undercarriage has eight solid rubber tyre wheels mounted on the gearboxes with four $7 \mathrm{kw}(9 \mathrm{hp})$ electric motors for continuous operation and has $24 \mathrm{kw}$ ( $32 \mathrm{hp}$ ) on peak through 30:1 gearboxes. The unit has independent braking systems, including two spring-applied electro-magnetic disc brakes and three piston stabilisers.

\subsection{Robot arm}

The robot arm on the robot consists of a lower two-stage, telescoping arm with an electric drive motor

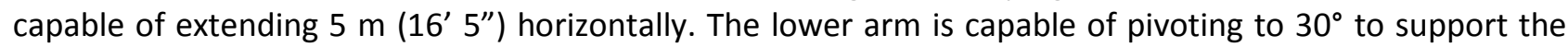
opening of the arm in $3.6 \mathrm{~m}$ (11' 9.7") back height. The upper arm is connected to the lower arm and is capable of pivoting to $135^{\circ}$ and extending to $10 \mathrm{~m}\left(32^{\prime} 10^{\prime \prime}\right)$. A stinger is attached to the lower arm to support the weight of the arms at their full extensions. The arm is shown at full extension in Figure 6.

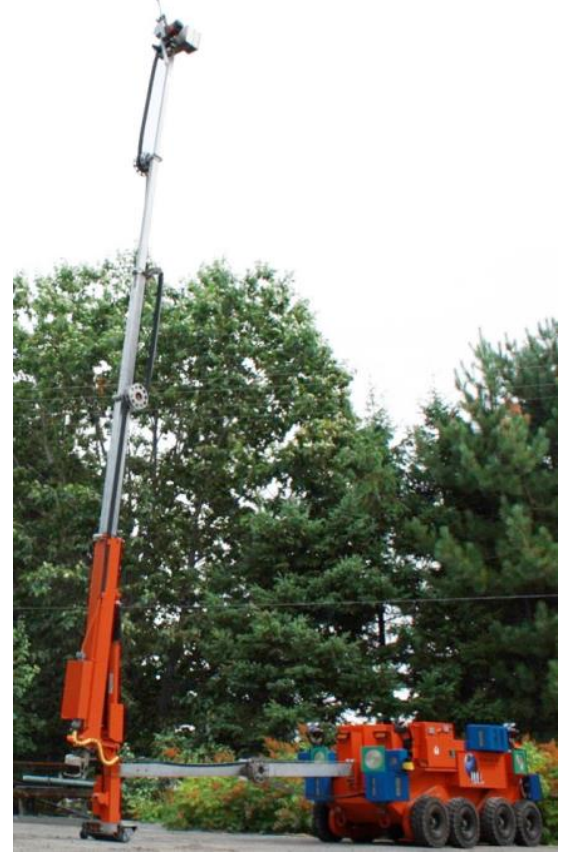

Figure 6 Hang-up telerobot with the lower and upper arm extended 
When the robot is reversed, two lower stingers can be extended to raise the rear of the robot so the upper

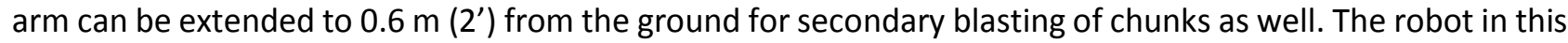
configuration is shown in Figure 7.

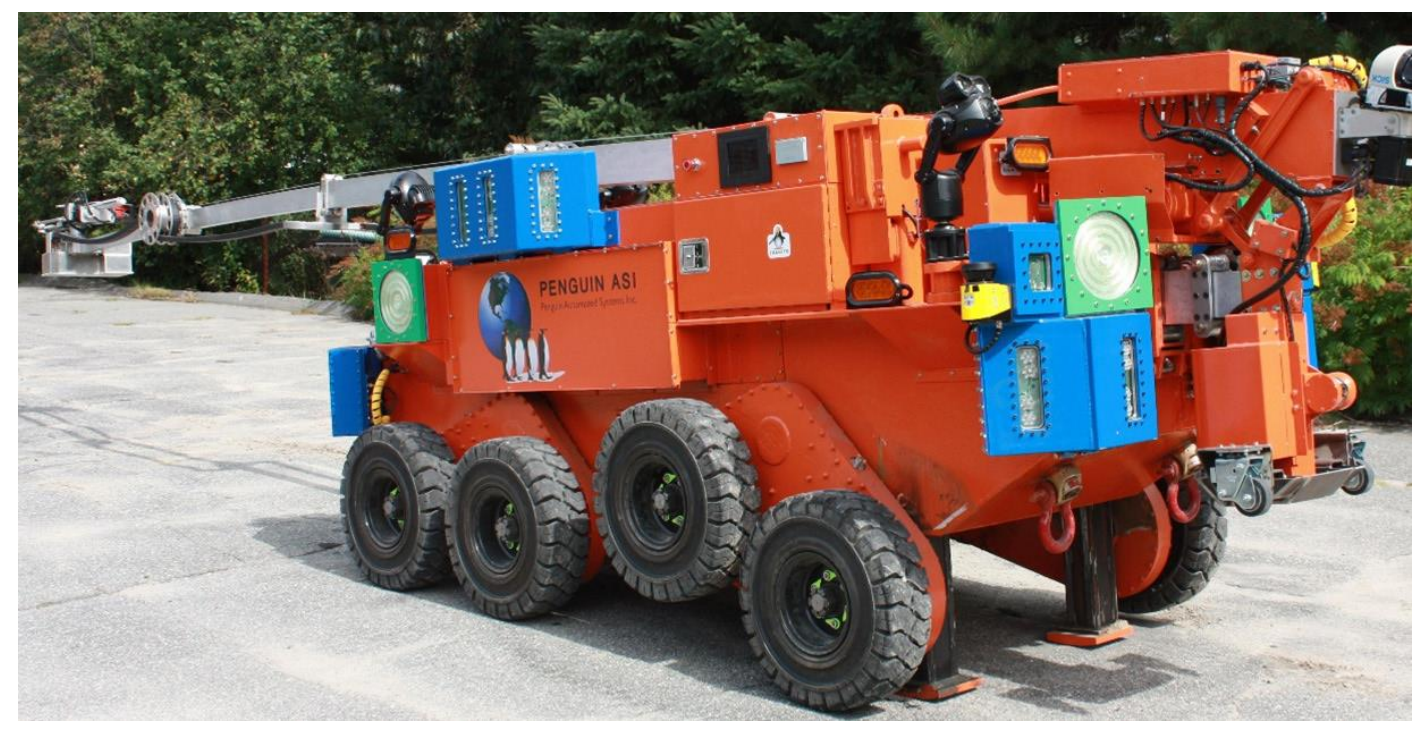

Figure 7 Hang-up telerobot reversed to drill and blast oversize

\subsection{End effector}

The robot system has an end effector capable of drilling, loading explosives and blasting in the rock hang-up at a precise location for hang-up breakage. The end effector uses an electric rock drill and bit selected to drill a hole $305 \mathrm{~mm}\left(12^{\prime \prime}\right)$ long and $34 \mathrm{~mm}\left(1.34^{\prime \prime}\right)$ in diameter. The drill is mounted on a table with a screw feed to thrust the drill into the rock. Once the hole is drilled, the table is rotated to thrust the explosives into the hole. The end effector holds a 3D camera system to allow the operator to track the progress of the drilling of the hole and the insertion of the explosives. Power and Ethernet at the end effector support the use of any other power tool.

Additional end effectors are under development now. These include the ability to glue the charge to the hang-up and a water cannon.

\subsection{Power system}

The robot and the multi-stage arm and end effector are all-electric. At the heart of the system are two lithium-ion phosphate batteries giving the robot an eight hour shift of operating time before charging. This power system allows the design of a high-reliability robot.

\subsection{System control and communications}

The Normet RBO truck with the telecommand/robot control system consists of many components. The most crucial system is the wireless networking system. Without the wireless system, this robot system would be impossible to build and operate. Penguin's proprietary Wireless Optical System (WOS) is the key component that allows this system to work. The WOS is capable of 100 megabytes per second to support the low latency requirements of the camera system, data system and scanning system needed to run the components of the Hang-up Assessment and Removal Robot System.

The WOS combines with a local wireless fidelity (WiFi) system to support long and short-range operation. The WOS is located on the front of the Normet RBO truck (Figure 8) and on all four sides of the robot as shown in Figure 9. The system shown has several light-emitting panels and light-receiving devices. Configuration of these panels is important to the full operation of the system. 


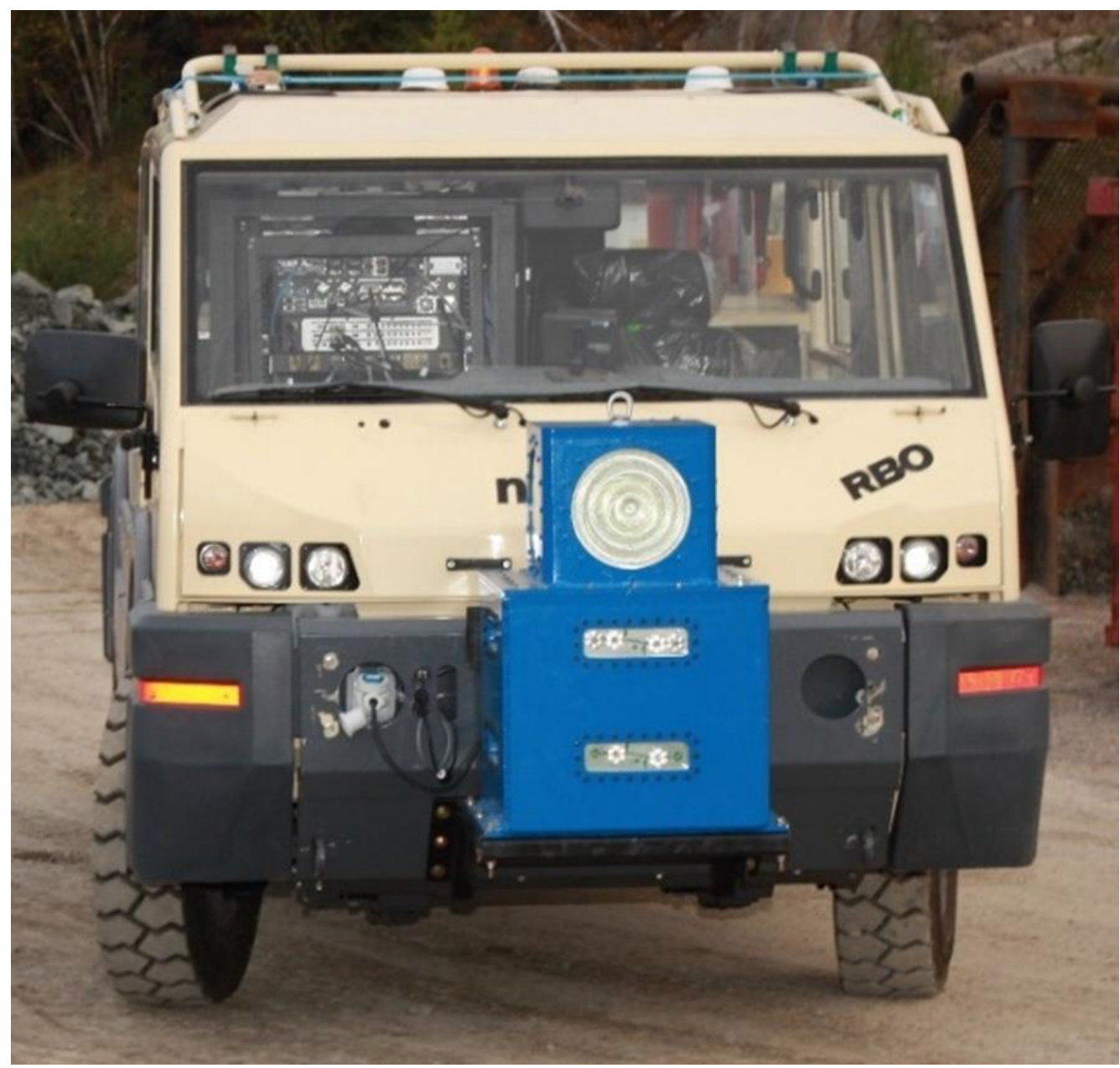

Figure 8 Optical communication system mounted on the RBO

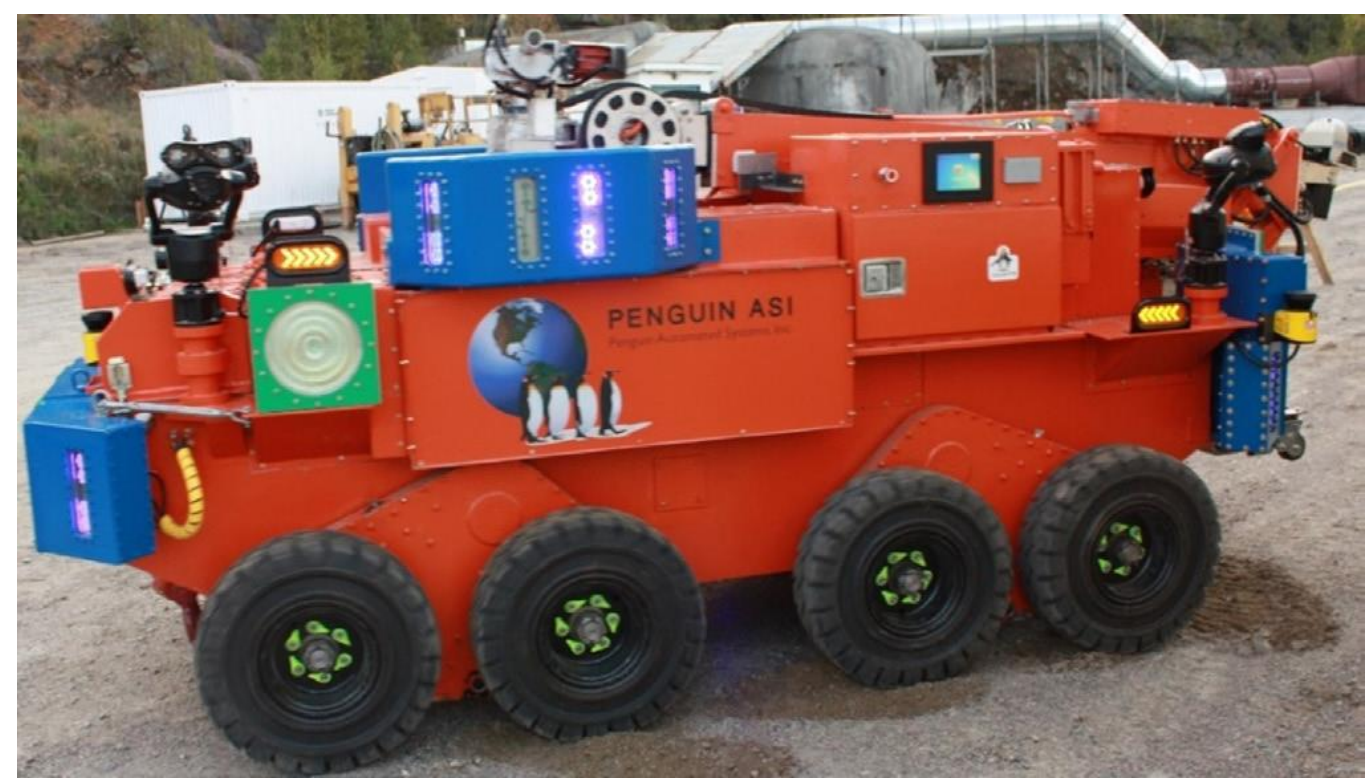

Figure 9 Optical communication system mounted on the telerobot 
The RBO telecommand centre has several devices for the operator interface as shown in Figure 10. These are:

- Multiple video system operation interface.

- A pair of multifunction joysticks.

- Computers mounted in a rack into the RBO.

The visualisation system accesses all cameras on-board and runs the proprietary machine software. Joysticks run all the drive and arm functions.

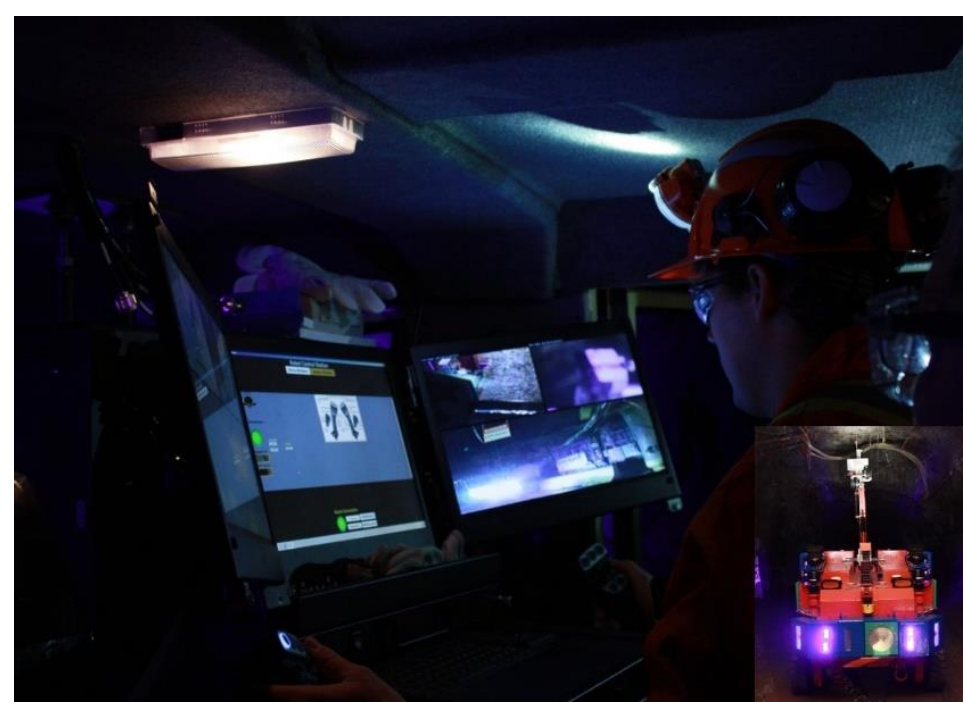

Figure 10 Telecommand centre

\subsection{Robot control system}

The robot control system is linked to a Normet RBO truck housing, a command centre via wireless optical and radio frequency networking. The system on board the robot consists of the following components:

- A hardened military grade industrial computer.

- A military grade positioning unit.

- Robot control system.

- Arm control system.

- Laser scanning system.

- Four pan, tilt, zoom (PZT) cameras.

- Two infrared drive cameras.

- On-board computer for local control.

- Vehicle network.

- Safety system.

\subsection{Robot computer and positioning system}

At the heart of the system is a hardened military/industrial computer platform. This system is linked to the underground positioning unit, cameras and sensors, and actuators in real time via Penguin's software control system. The two units are shown in Figure 11. 

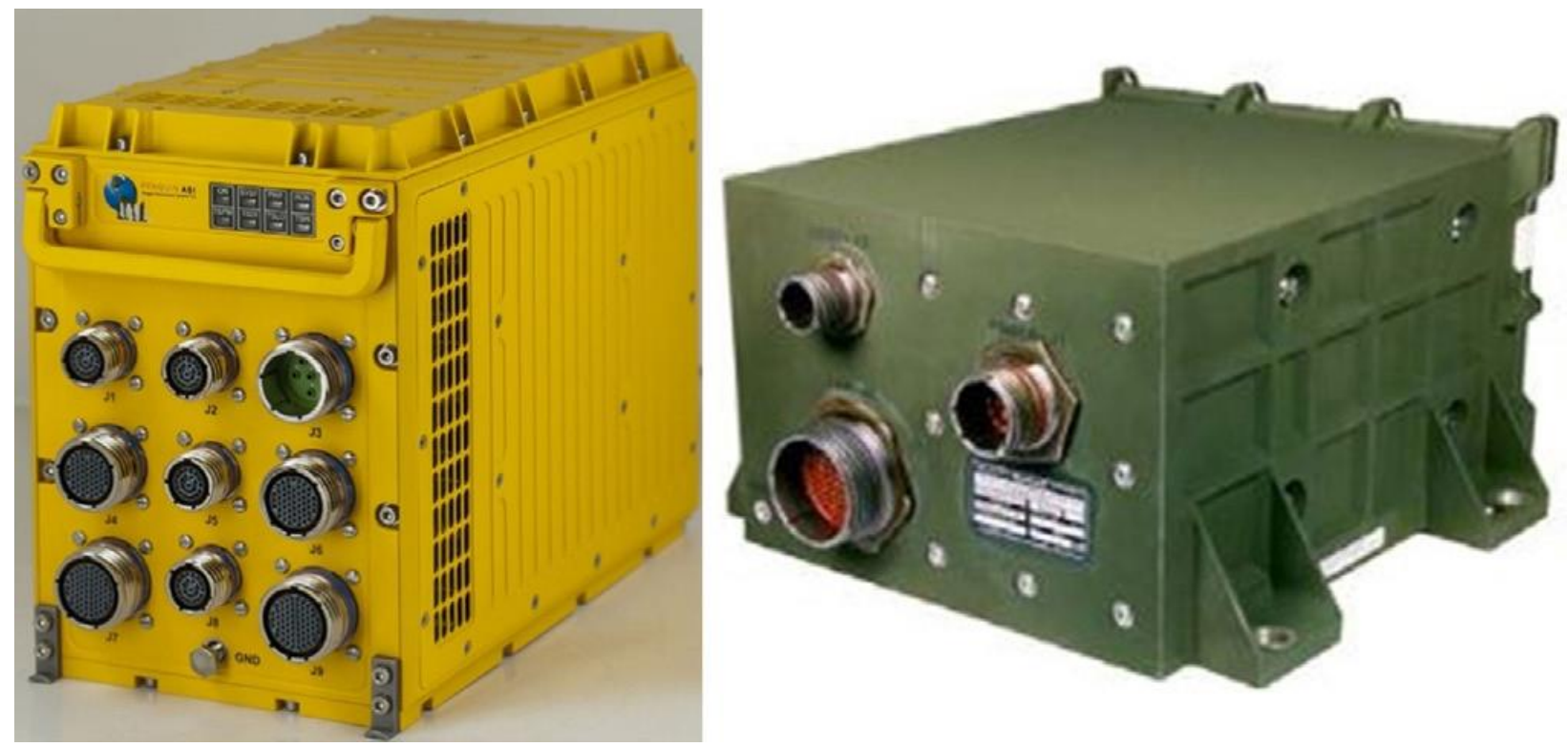

Figure 11 Penguin on-board computational platform and positioning unit

\section{$4 \quad$ System testing}

Penguin used the Northern Centre for Advanced Technology (NORCAT) test mine for testing the Hang-up Assessment and Removal Telerobot in April 2016. This test program covered the various aspects of the machine system. The main tests undertaken were loading/unloading, position, communication, safety system, teleoperation and underground operation. The test program emulated the Andina testing requirements prior to shipping the system to Codelco.

\subsection{Position}

The key to this telerobot is its ability to geospatially locate itself in an underground situation. Therefore, this testing was quite extensive. The hang-up assessment and removal telerobot has been conceived as a mathematical machine equivalent to a mobile $\mathrm{CNC}$ mill or lathe in manufacturing. The hang-up removal robot needs to be providing positional information as the robot moves throughout the whole machine to the drill bit.

The testing consisted of:

- Initial point.

- Inertial measurement unit initialisation.

- Locate angle measurements and motors.

- Scanning system.

- Machine model.

- Virtual hang-up removal.

The use of this robot requires the establishment of an initial point either on-surface or underground.

\subsection{Communication}

Initial testing of the system included field testing with line-of-sight and non-line-of-sight locations. The combined use of radio (WiFi) and optical (light fidelity - LiFi) networking with Ethernet successfully increased the range and capacity of the teleoperation. 


\subsection{Teleoperation}

The Hang-up Assessment and Removal Robotic System was tested underground in the NORCAT research mine in Sudbury. The system, while not in an actual drawpoint, did run through each test prior to shipping to Codelco for final drawpoint testing. The unit ran through the tests outlined here:

- Connection test

- Loss of connection

- Latency response

- Robot set-up

○ Unloading/loading

○ Positioning

- Scanning

- Arm deployment

- Lower arm angle

- Lower arm extension

- Upper arm unfold

- Upper arm extension

- End effector

- Initialisation

○ Drill

- Explosives

- Leg wire

\subsection{Underground operation}

Connection test - During these tests, network connection was verified underground between the Normet RBO truck command centre and the hang-up robot for both the radio and optical network. Loss of connection was simulated along with latency response to ensure safe operation and the ability to do the work underground.

Robot set-up - The teleoperated system was then tested to ensure all functions to achieve the final result of a prepared loaded charge ready for blasting could be completed. The scanning step was completed successfully and then the arm was deployed using several simulated configurations with the final tests of hole drilling, explosives insertion and backing the robot away pulling the explosive detonation wire to a safe location for personnel to work safely with it in blasting the area.

Underground operation - The successful testing of the hang-up robot in underground operation is a significant milestone. This mobile robot represents the first equivalent of a ruggedised manufacturing robot on a moving platform that can be operated over any network with sufficient capacity where a need for accurate placement of a work element (drill, charge, etc.) can be done without the need for a person to be there. This unit, and others like it, will begin to be the norm in mining and construction in difficult environments in the future. 


\section{Summary}

A prototype of a new mining robot system has been created to allow miners to safely remove hang-ups and rock blockages from mines, in particular caving type mines.

Key features include:

- Teleremote operator control of the system to ensure workers can stay safe while removing rock blockages such as hang-ups and oversize.

- The system is capable of providing a 3D image of the hang-up without the operator needing to expose themselves to potential falling rock hazards.

- A robot arm mounted on the body of the robot is capable of reaching $3 \mathrm{~m}\left(9^{\prime} 10^{\prime \prime}\right)$ horizontally and $10 \mathrm{~m}\left(32^{\prime} 10^{\prime \prime}\right)$ vertically.

- An end effector capable of drilling a hole and loading the hole in the precise position required.

These features have been tested underground in Sudbury. Figure 12 shows the unit in an underground drift during commissioning and testing.

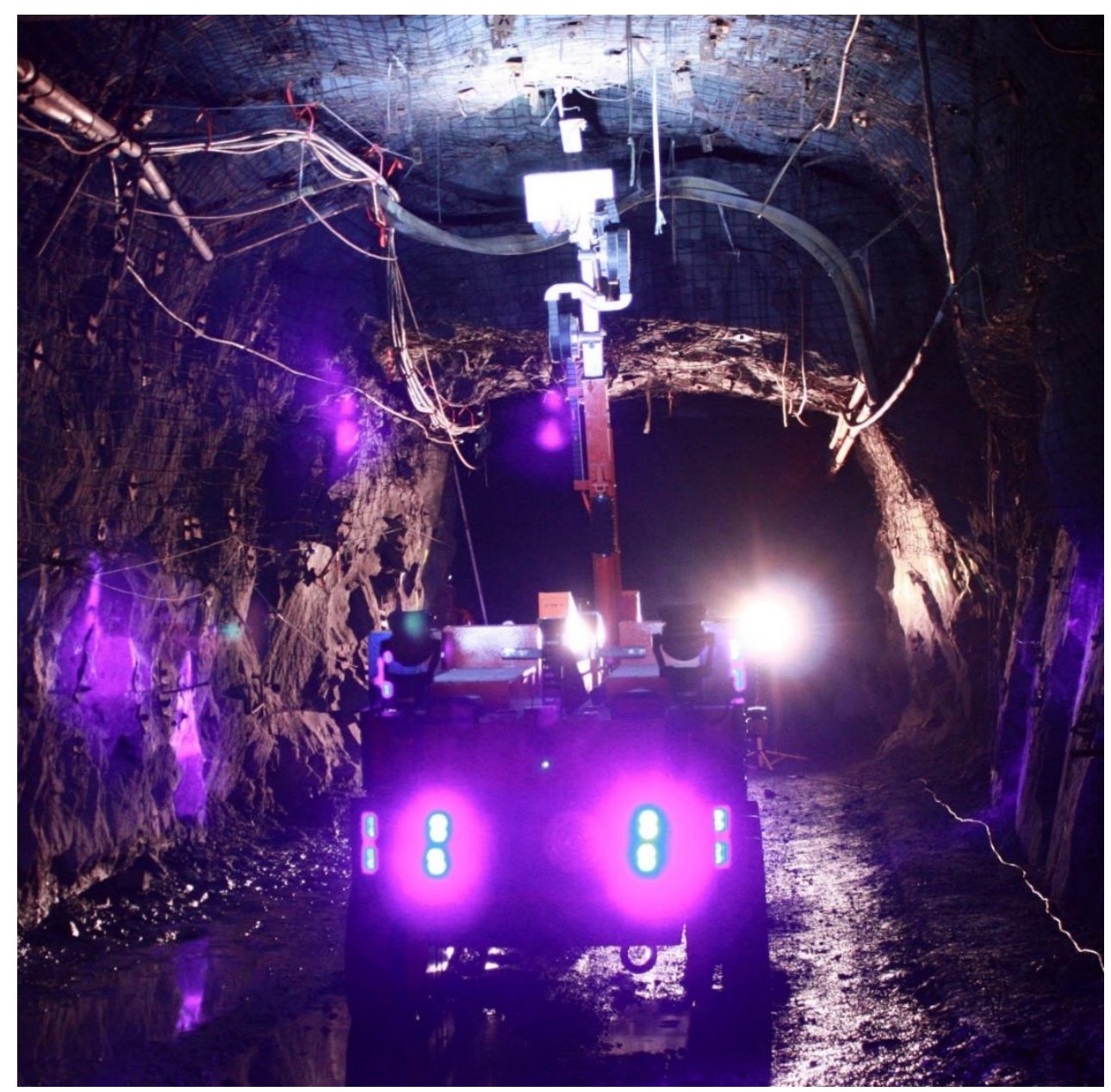

Figure 12 Hang-up telerobot drilling a hole at the research mine

\section{References}

Chitombo, GP 2010, 'Cave mining - 16 years after Laubscher's 1994 paper 'Cave mining - state of the art”, in Y Potvin (ed.), Proceedings of the Second International Symposium on Block and Sublevel Caving, Australian Centre for Geomechanics, Perth, pp. 45-61.

Laubscher, DH 1994, 'Cave mining - the state of the art', Journal of the South African Institute of Mining and Metallurgy, vol. 94, no. 10 , pp. 279-293. 
